\title{
Multiplexed SQUID Array for Non-Destructive Evaluation of Aircraft Structures
}

\author{
Hans-Joachim Krause, Stefan Gärtner, Norbert Wolters, Rainer Hohmann, Walter Wolf, \\ Jürgen Schubert, Willi Zander, and Yi Zhang \\ Marc v. Kreutzbruck and Michael Mück
}

\begin{abstract}
SQUID sensors offer a significant advantage for eddy-current $(E C)$ testing of aircraft components for material flaws hidden deeply in the tested structure. However, the requirement to take maps of the magnetic field, usually by meander-shaped scans, leads to unacceptably long measurement times. Due to their inductive coupling to a tank circuit, several rf SQUID sensors may be read out sequentially by selectively coupling to their tank circuits, using only one electronics with a multiplexer [1]. The multiplexed operation of three planar HTS rf SQUID gradiometers with one electronics and one cable is shown, demonstrating the advantage of lower liquid nitrogen boil-off. Independent operation and switching is confirmed using local coil excitation of the individual SQUIDs. We report on the implementation of two multiplexed SQUID sensors in conjunction with an EC excitation and lock-in readout at unshielded laboratory environment. Scanning is performed while continuously switching the operating SQUID, thus obtaining two traces simultaneously. The applicability to EC testing of riveted sections of aircraft fuselage is discussed.
\end{abstract}

Index Terms-Arrays, Multiplexing, Nondestructive Testing, SQUIDs.

\section{INTRODUCTION}

$\mathrm{M}$ ultichannel SQUID systems are becoming increasingly popular not only in the field of biomagnetism but also in non-destructive evaluation of materials. More information on the magnetic field distribution around the source may synchronously be collected, paving the road towards more sophisticated signal analysis and eventually solving the inverse problem [2]. Generally, many system parameters scale approximately linearly with the number of SQUID channels: cost, size, power consumption, adjustment effort, number of cables from the warm to the cold end and thus coolant evaporation rate, due to thermal conduction into the cryostat. With growing number of SQUID channels, it becomes increasingly important to introduce multiplexing, i.e. sharing some parts of the system among several channels.

Some interesting ideas have been published regarding

Manuscript received September 18, 2000. This work was supported in part by the German BMBF under Grant No. 13 N 7429/0.

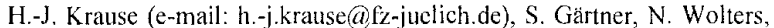
R. Hohmann, W. Wolf, J. Schubert, W. Zander, and Y. Zhang are with the Institut für Schicht- und Ionentechnik (ISI), Forschungszentrum Jülich GmbH, D 52425 Jülich, Germany.

M. v. Kreutzbruck and M. Mück are with the Institut für Angewandte Physik, Universität Giessen, D-35392 Giessen, Germany.
SQUID multiplexing [3]. Furukawa and Shirae [4] suggested a multichannel system where the sum of all channels modulates yet another SQUID. Only this readout SQUID requires a transmission line and a preamplifier, whereas the signals from the individual channels are separated by phase detectors. However, each SQUID requires its own feedback coil connection.

Radio frequency (rf) SQUIDs are particularly well suited for multiplexing due to the inductive readout concept using an if demodulation of their response. Some time ago we demonstrated a three channel rf SQUID system [I] in which several rf SQUIDs were read out serially by a single $r f$ SQUID electronics using one transmission line. The selection of the individual SQUID channel was accomplished by using different $\mathrm{rf}$ pumping frequencies for each single SQUID and by tuning the $\mathrm{rf}$-bias frequency to the resonance of the corresponding tank circuit. In a sense, the detection scheme is comparable to the principle of a heterodyne radio receiver. Different SQUID sensors correspond to different radio stations which are selected by tuning the receiver's local oscillator to their frequency.

In this work, we describe a multiplexed SQUID array consisting of three planar HTS rf SQUID gradiometers for eddy current testing of rivet joints in aircraft fuselage. Due to their wide dynamic range, SQUID sensors are well suited for eddy-current (EC) testing of aircraft components, and -because of their high field sensitivity at low frequencies are especially useful to detect material flaws hidden deeply inside highly conductive samples [5]. Certain NDE procedures require two-dimensional magnetic field maps, usually taken by meander-shaped scans. The time required for such measurements can be shortened substantially by using a one- or two-dimensional array of SQUIDs. For example, the implementation of a linear array with several SQUIDs distributed over 20 to $40 \mathrm{~mm}$ allows to test a rivet row in one single scan.

\section{MUI,TIPLEXING CONCEPT}

\section{A. Fast switching between channels}

For biomagnetic applications, the interesting signal frequencies are typically less than $200 \mathrm{~Hz}$. In this case, quasisimultaneous read-out of all SQUID channels can be achieved. Some time ago we [1] realized the following concept which is applicable in the limit that the signal 
frequencies to be measured are small compared to the multiplex frequency, and of which Fig. 1 shows the setup schematically. Three LTS if SQUIDs are read out using one SQUID electronics, with multiplexed individual oscillators and feedback integrators.

Since each SQUID is threaded by a different flux, the current through each feedback coil must be different. When each SQUID is operated only a fraction of the time, the value of its flux must be stored and applied again to the feedback coil prior to the next operation cycle. Thus, each SQUID has its own integrator which stores the channel's dc value and is multiplexed synchronously. That means that as long as any one SQUID is operating, its integrator is switched to (all) the feedback coils. Thus, the fast switching scheme is applicable only if the flux change in each channel is smaller than $\Phi_{0} / 4$ during one multiplex cycle. A multiplex cycle denotes the time after which any one channel is read out again, it is equal to the number of channels divided by the multiplex frequency.

\section{B. Slow switching between channels}

In case the SQUID array is to be used as a sensor in an eddy current testing system, a simplified multiplexing scheme may be used. Since the dc value of the flux measured by each channel is unimportant, it doesn't have to be stored in separate integrators. Only the amplitude and phase of the response of each SQUID at the excitation frequency is of interest. Provided that the measurement time for each channel

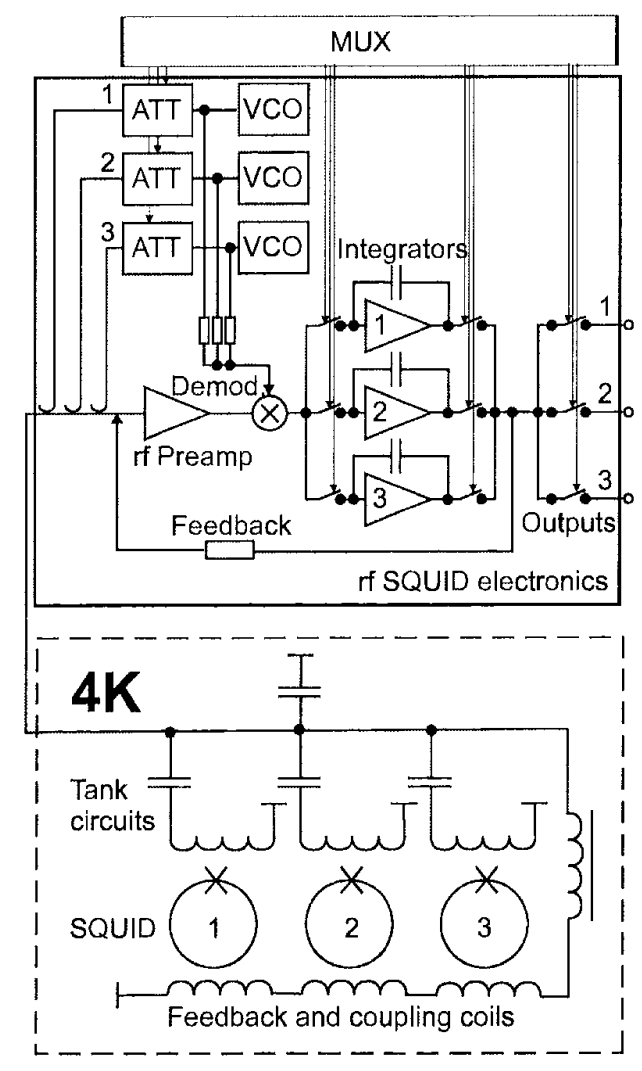

Fig. 1. Scheme of the multiplexed three channel system [from 1].
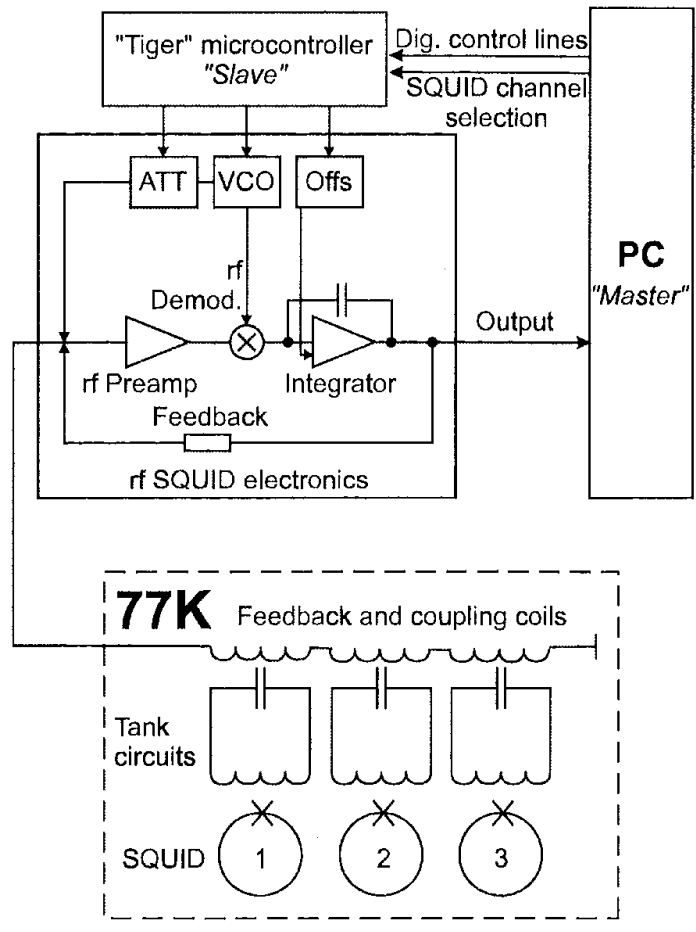

Fig. 2. Scheme of the slow switching concept used in this work.

is long enough to cover a few periods of the excitation, lockin determination of in-phase and quadrature component of the response is feasible before switching to the next channel. Fig. 2 shows the multiplexing scheme for three rf SQUIDs in that case. The NDE system with SQUID arrays presented in this paper was realized using this scheme.

\section{EXPERIMENTAL}

\section{A. System configuration}

Fig. 3 shows the components of a prototype realized following the scheme shown in Fig. 2. As SQUID sensors, planar double hole HTS rf gradiometers were used [6]. They were fabricated from laser-ablated $\mathrm{Y}-\mathrm{Ba}-\mathrm{Cu}-\mathrm{O}$ films on $\mathrm{La}-$ Al-O substrates using step-edge Josephson junctions, and had a $3.7 \mathrm{~mm}$ long baseline and $2.5 \mathrm{~mm}$ washer diameter. The field-to-flux coefficient of the gradiometers was approx. $34 \mathrm{nT} /\left(\Phi_{0} \mathrm{~cm}\right)$, with a white flux noise of 70 to $130 \mu \Phi_{0} / \mathrm{JHz}$ and a balance of better than 130 .

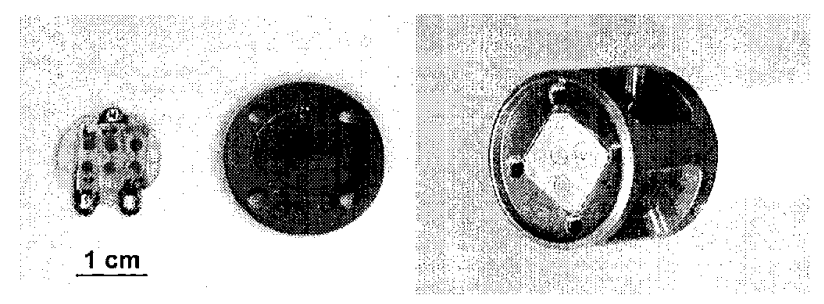

Fig. 3. Photographs of the components of a first prototype. From left to right: Printed circuit board with three tank circuits, three SQUID substrates placed upon them, lid with pockets for SQUIDs and capacitors, and holder with the three coupling coils. 


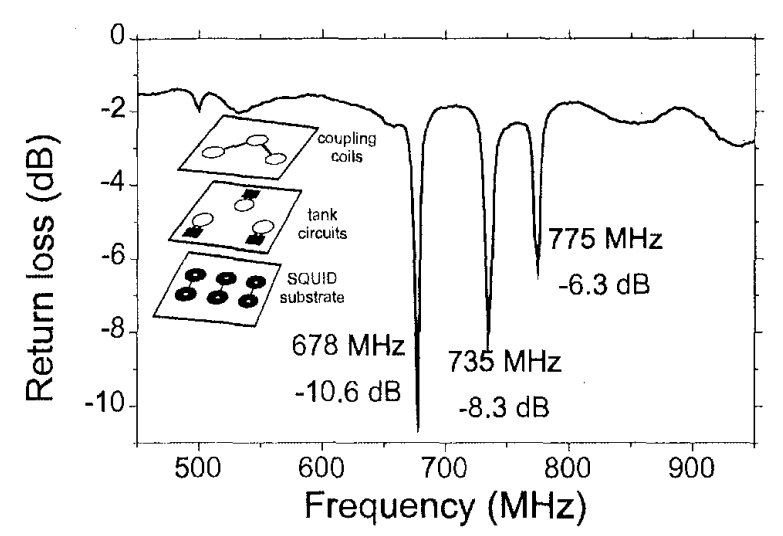

Fig. 4. Optimized return loss of the three-SQUID system with all three tank circuits on one printed circuit board.

In order to couple the SQUIDs to the read-out electronics, we fabricated three inductively coupled, galvanically isolated tank circuits [7] on a printed circuit board. A second circuit board with three coupling coils connected in series was placed directly above the tank circuits.

\section{B. Optimization of the rf coupling}

The if coupling between coupling coil and tank circuit and the matching to the $50 \Omega$ transmission line was adjusted by varying the diameter of the coupling coil from $0.9 \mathrm{~mm}$ to $2.2 \mathrm{~mm}$. A coil diameter of $1.7 \mathrm{~mm}$ was found to yield optimum results. The coupling between tank circuit and SQUID was optimized by varying the distance between each individual SQUID and its tank circuit from 0 to $0.4 \mathrm{~mm}$, using $0.1 \mathrm{~mm}$ Vespel foils as spacers. Fig. 4 shows the resulting optimum return loss. The spacings were adjusted to $0.1,0$ and $0.3 \mathrm{~mm}$ for SQUIDs $\# 1,2,3$ and yielded return losses of $-6.3,-10.6$ and $-8.3 \mathrm{~dB}$, respectively. These values are somewhat low. Return losses of more than $-20 \mathrm{~dB}$ are considered optimum for single rf SQUID systems.

\section{Determination of cross-talk between channels}

The cross-talk between SQUID channels was determined by placing a small plate with three solenoids ( $1 \mathrm{~mm}$ dia., 3 windings of $0.1 \mathrm{~mm}$ copper wire) close to the SQUIDs. Coil $\# \mathrm{i}(\mathrm{i}=1,2,3)$ was placed directly underneath one loop of SQUID \#i. Due to substrate and housing, the distance was approx. $2 \mathrm{~mm}$. Thus, a quasi-local excitation of each SQUID was realized. Table I displays the responses of each SQUID to each of the three coils, when a small ac excitation current was applied to it. The diagonal elements dominate in the table, as expected. Thus, the main response is received from the SQUID located right next to each of the coils.

TABLE I

CROSS-TALK BETWEEN CHANNELS

\begin{tabular}{lccc}
\hline $\begin{array}{l}\text { Active } \\
\text { excitation coil }\end{array}$ & $\begin{array}{c}\text { Output voltage of SQUID (mV) } \\
\text { SQUID \#1 } \\
\text { (left) }\end{array}$ & $\begin{array}{c}\text { SQUID \#2 } \\
\text { (middle) }\end{array}$ & $\begin{array}{c}\text { SQUID \#3 } \\
\text { (right) }\end{array}$ \\
\hline Coil \#1 & 350 & 75 & 0 \\
Coil \#2 & 75 & 500 & 95 \\
Coil \#3 & 0 & 65 & 850 \\
\hline
\end{tabular}

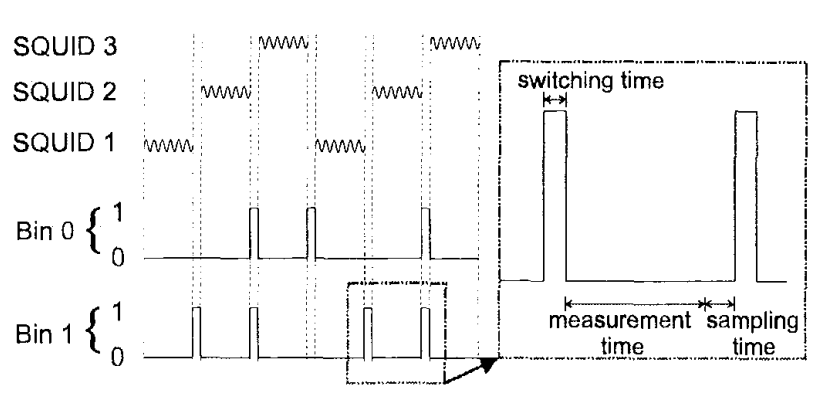

Fig. 5. Time diagram of multiplexing of the three-SQUID system.

Next neighbor SQUIDs respond typically with 8 to $20 \%$. We attribute this effect to alignment errors of the manually wound coils and to imbalance of the planar gradiometer. In order to check the possibility that the off-diagonal responses arise from spurious responses from the two other SQUIDs not selected by VCO frequency, the selected SQUID substrate was removed. No SQUID signal was observed in that case. It was not possible to couple to any SQUID from a neighboring tank circuit. Coupling could only be achieved with the tank circuit lying directly above a SQUID.

\section{Multiplexing}

As readout electronics, we used a V3.5 HTS rf SQUID electronics with a "Tiger" microcontroller, available from JSQ [8]. The controller was modified such that it works as a slave: its state is switched by two digital control lines -.. see Fig. 2. The code "1", binary "01", means: open the fluxlocked loop (FLL) and adjust VCO, attenuator and offset to the previously saved working point of SQUID \#1. The code "2", binary "10", means: open the FLL and adjust to the working point of SQUID \#2; code " 3 ": adjust to \#3. Code " 0 " means: close the FLL and measure. Fig. 5 schematically displays the sent codes and received SQUID responses during two complete cycles. The SQUID selection and timing responsibility lies completely at the master $\mathrm{PC}$.

\section{E. Multiplexed Scanning and Lock-in Detection.}

For eddy current measurements, the SQUID electronics output is connected to a lock-in amplifier. Fig. 6 shows the response of two multiplexed SQUID gradiometers placed in the center of a $90 \mathrm{~mm}$ dia. excitation coil driven at $468 \mathrm{~Hz}$.

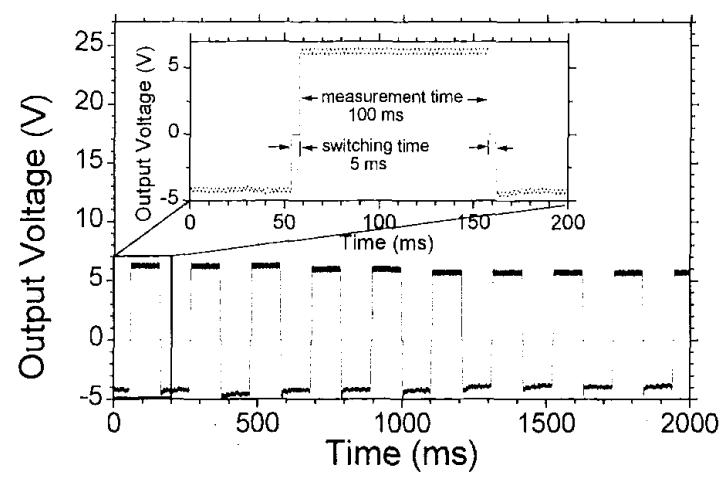

Fig. 6. Time trace of the SQUID electronics output during multiplexed operation of two SQUIDs. 


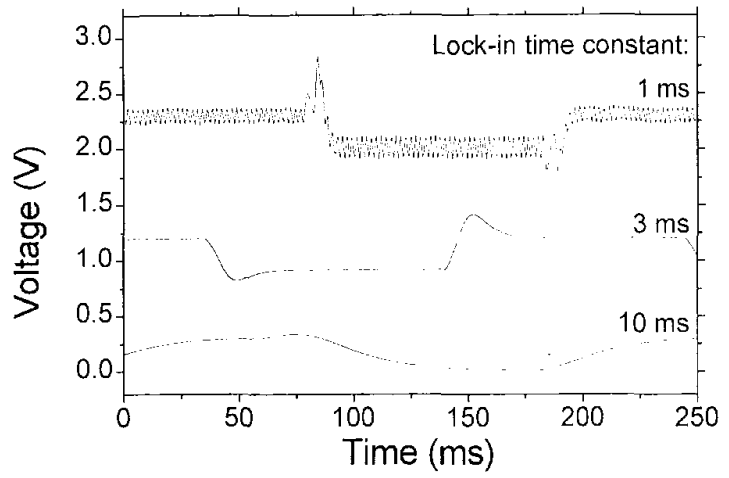

Fig. 7. Time trace of the lock-in amplifier's output during multiplexed operation of two SQUIDs. The three curves were taken with three different lock-in time constants 10,3 and $1 \mathrm{~ms}$. The time scale is not synchronized with the control, therefore switching points vary. For clarity, the curves are displayed with offset 0,1 and $2 \mathrm{~V}$, respectively.

Underneath the SQUID array and the excitation coil, a $1.5 \mathrm{~mm}$ thick aluminum plate with a sawcut flaw was scanned. Fig. 7 shows the in-phase output of the lock-in amplifier at a position where one SQUID is closer to the flaw than the other, thus yielding a stronger eddy current response. The lock-in-detected SQUID signals are clearly different for both channels. The time constant has to be selected sufficiently large to suppress the eddy current frequency ( $3 \mathrm{~ms}$ in our case). The measurement time can then be reduced to about five to seven times the time constant so that relaxation of the lock-in is obtained.

\section{IMPROVED SYSTEM CONFIGURATION}

As discussed in Section III B, the relatively low rf return losses imply that the coupling to the SQUIDs is not sufficient. For this reason, a stable multiplexed operation of three SQUIDs was not possible with the configuration discussed above. Assuming that the tank circuits were placed too close to each other which might lead to mutual damping, we decided to check the dependence of the return loss on separation of the coils and cut the tank circuit board into three pieces. It turned out that from this mere cutting, the coupling between tank circuit and SQUID improved considerably.

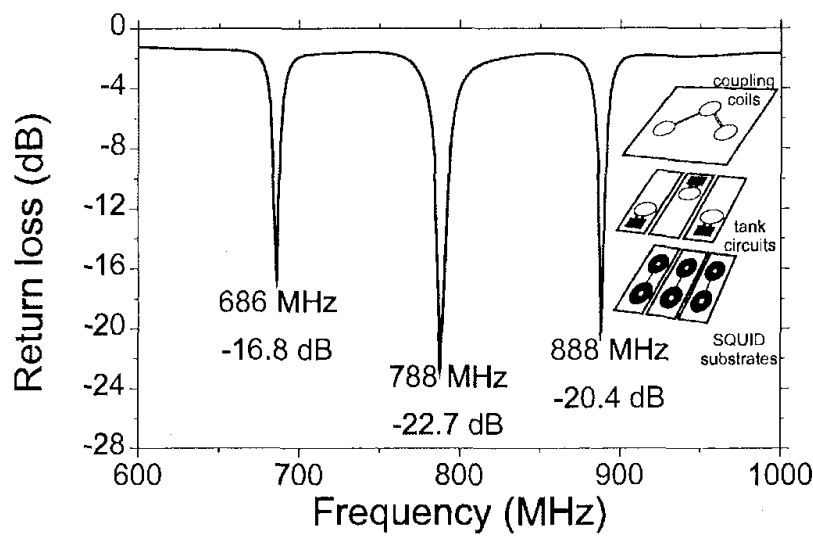

Fig, 8. Optimized return loss of the three-SQUID system with tank circuits on separate boards.
Return losses went up by about a factor of two (see Fig. 8), even when placing the tank cicuit boards right next to each other. This may be explained by the introduction of two boundaries, from $\varepsilon \sim 5$ of the circuit board material at $800 \mathrm{MHz}$ to $\varepsilon=1$ in air. When varying the distance between substrates from 0 to $4 \mathrm{~mm}$, return losses and SQUID noise remained almost constant. With this configuration, stable multiplexed operation of three SQUIDs was achieved.

\section{CONCLUSION}

The sequentially multiplexed operation of an array of three planar HTS rf SQUID gradiometers with one electronics by selectively coupling to their tank circuits was shown. As the SQUID array is to be used for eddy current testing in NDE of aircraft, a simplified modulation setup without keeping track of the feedback flux could be realized, using just one integrator. Independent operation and switching was confirmed using local coil excitation of the individual SQUIDs. An eddy current measurement with multiplexed SQUID sensors and lock-in readout was demonstrated. By introducing air gaps between tank circuits, the coupling and thus the operation stability was improved considerably. Practical advantages for application in aircraft testing include the feasibility of scanning a rivet row with 6 to 9 parallel SQUIDs simultaneously and thus obtaining an eddy current map without meander scanning. Advantage over conventional multichannel SQUID systems are reduction of the number of electronics, reduced power and coolant consumption. Future work will be devoted to the development of a prototype system for testing rivet rows in aircraft fuselage.

\section{ACKNOWLEDGMENT}

The authors thank M. Junger (Rohmann GmbH), W.-B. Klemmt (EADS Airbus), W. Becker (Lufthansa Technik $\mathrm{AG}$ ), and A. Binneberg (ILK Dresden) for many stimulating discussions and for providing aircraft samples. Special thanks to P. David (University of Bonn) for numerous helpful discussions, his continuos interest and support.

\section{REFERENCLS}

[1] M. Miick, "A Three Channel SQUID-system with Multiplexed Readout," IEEE Trans. Mag., vol. 27, pp. 2986-2989, 1991.

[2] J.P. Wikswo, "The magnetic inverse problem for NDE," in $S Q U I D$ sensors: fundamentals, fabrication and applications, H. Weinstock, Ed. Dordrecht: Kluwer, 1996, pp. 629-696.

[3] M. Mück, "Radio frequency SQUIDs and their applications," in Applications of superconductivity, H. Weinstock, Ed. Dordrecht: Kluwer, 2000, in press.

[4] H. Furukawa, K. Shirae, "Multichannel dc SQUID system," Jap. J, Appl. Phys, vol. 28, pp. L456-458, 1989.

[5] M. v. Kreutzbruck, K. Allwcins, T. Rühl, M. Mück, C. Heiden, H.-J. Krause, R. Hohmann, "Defect detection and classification using a SQUID based multiple frequency eddy current NDI: system," IEEE Trans. Appl. Supercond, this issue.

[6] Y. Zhang et al., "Planar HTS Gradiometers with Large Baseline," IEEE Trans. Appl. Supercond., vol. 7, pp. 2866-2869, June 1997.

[7] D.F. He, X.H. Zeng, H.-J. Krause, H. Soltner, F. Rüders, and Y. Zhang, "rf SQUIDs operating at $77 \mathrm{~K}$ with $1 \mathrm{GHz}$ lumped-element tank circuits," Appl. Phys. Lett., vol. 72, pp. 969-971, January 1998.

[8] Jülicher SQUID GmbH (JSQ), http: Iwww jsquid.com. 